\title{
Emulators for the nonlinear matter power spectrum beyond $\Lambda \mathrm{CDM}$
}

\author{
Hans A. Winther $\odot,{ }^{1,2}$ Santiago Casas, ${ }^{3}$ Marco Baldi, ${ }^{4,5,6}$ Kazuya Koyama, ${ }^{1}$ Baojiu Li, ${ }^{7}$ \\ Lucas Lombriser, ${ }^{8}$ and Gong-Bo Zhao ${ }^{9,1}$ \\ ${ }^{1}$ Institute of Cosmology \& Gravitation, University of Portsmouth, Portsmouth, \\ Hampshire PO1 3FX, United Kingdom \\ ${ }^{2}$ Institute of Theoretical Astrophysics, University of Oslo, 0315 Oslo, Norway \\ ${ }^{3}$ AIM, CEA, CNRS, Université Paris-Saclay, Université Paris Diderot, Sorbonne Paris Cité, \\ F-91191 Gif-sur-Yvette, France \\ ${ }^{4}$ Dipartimento di Fisica e Astronomia, Alma Mater Studiorum Universit di Bologna, \\ viale Berti Pichat, 6/2, I-40127 Bologna, Italy \\ ${ }^{5}$ INAF_-Osservatorio Astronomico di Bologna, via Ranzani 1, I-40127 Bologna, Italy \\ ${ }^{6}$ INFN-Sezione di Bologna, viale Berti Pichat 6/2, I-40127 Bologna, Italy \\ ${ }^{7}$ Institute for Computational Cosmology, Department of Physics, Durham University, \\ Durham DH1 3LE, United Kingdom \\ ${ }^{8}$ Departement de Physique Thorique, Universit de Genve, 24 quai Ernest Ansermet, \\ 1211 Genve 4, Switzerland \\ ${ }^{9}$ National Astronomy Observatories, Chinese Academy of Science, Beijing, 100012, \\ People's Republic of China
}

(Received 9 May 2019; published 23 December 2019)

\begin{abstract}
Accurate predictions for the nonlinear matter power spectrum are needed to confront theory with observations in current and near future weak-lensing and galaxy clustering surveys. We propose a computationally cheap method to create an emulator for modified gravity models by utilizing existing emulators for $\Lambda \mathrm{CDM}$. Using a suite of $N$-body simulations, we construct a fitting function for the enhancement of both the linear and nonlinear matter power spectrum in the commonly studied Hu-Sawicki $f(R)$ gravity model valid for wave numbers $k \lesssim 5-10 h \mathrm{Mpc}^{-1}$ and redshifts $z \lesssim 3$. We show that the cosmology dependence of this enhancement is relatively weak so that our fit, using simulations coming from only one cosmology, can be used to get accurate predictions for other cosmological parameters. We also show that the cosmology dependence can, if needed, be included by using linear theory, approximate $\mathrm{N}$-body simulations (such as comoving lagrangian acceleration) and semianalytical tools like the halo model. Our final fit can easily be combined with any emulator or semianalytical models for the nonlinear $\Lambda \mathrm{CDM}$ power spectrum to accurately, and quickly, produce a nonlinear power spectrum for this particular modified gravity model. The method we use can be applied to fairly cheaply construct an emulator for other modified gravity models. As an application of our fitting formula, we use it to compute Fisher forecasts for how well galaxy clustering and weak lensing in a Euclid-like survey will be at constraining modifications of gravity.
\end{abstract}

DOI: 10.1103/PhysRevD.100.123540

\section{INTRODUCTION}

One of the objectives of many next-generation surveys such as Euclid [1] and LSST [2] is to look for and constrain any deviations from the predictions of general relativity (GR). Modifications of gravity have been studied quite extensively over the last decade (see, e.g., Refs. $[3,4]$ and references within). Such modifications, when they are in agreement with local and astrophysical tests of gravity, usually reduce to having most of their interesting effects in the nonlinear regime of structure formation.

Testing such models and extracting the maximum information that is contained in the data gathered from current and future galaxy and weak-lensing surveys require us to include nonlinear scales. This requires theoretical predictions for the matter power spectrum on these scales.

Currently, this is either done using semianalytical predictions and/or fits like HALOFIT $[5,6]$ and HMCODE $[7,8]$ (which is implemented in commonly used Boltzmann codes such as CAMB [9] and CLASS [10]) or using an emulator such as CosmicEmu [11-13] and Euclidemulator [14]. An emulator is constructed by performing a large number of $\mathrm{N}$ body simulations in the parameter space and then performing an interpolation to obtain the power spectrum for any parameter combination of interest. This can be quite expensive to make, but once it is created, it can provide nonlinear spectra (usually from linear spectra) almost for free. 
For $\Lambda \mathrm{CDM}$, both of the approaches above have been adopted and used to provide constraints from observations. For the case of modified gravity models, the only fit provided so far is MGHALOFIT $[15,16]$, which has a modified HALOFIT that was calibrated using $N$-body simulation data to the Hu-Sawicki model [17]. For a coupled dark energy model, a fitting function was developed in Ref. [18] and was used to forecast the constraints on the coupling parameter $\beta$. To be able to derive constraints, or to provide forecasts for how well future experiments will constrain deviations from GR, we need to have an accurate model for the nonlinear matter power spectrum. This generally has to be derived on a model-by-model basis.

However, very recently, an interesting semianalytical method based on the halo model and nonlinear perturbation theory was proposed in Ref. [19] and shown to be able to get $1 \%-3 \%$ accuracy out to $k \sim 1 h \mathrm{Mpc}^{-1}$.

In this paper, we will consider the Hu-Sawicki model as this is a representative model when it comes to using cosmology and astrophysics to constrain deviations from GR. Our aim is to provide the community with a precise fitting function for this model that can be used for example for making forecasts for future surveys. Instead of providing a fit for $P_{f(R)}$ directly, we present a fitting function for the enhancement $P_{f(R)} / P_{\Lambda \mathrm{CDM}}$ (both for the linear and nonlinear power spectrum) as function of scale, redshift, and the model parameter $f_{R 0}$ which controls the size of the deviations from GR (GR is recovered as $f_{R 0} \rightarrow 0$ ). This enhancement, as we will show, has a fairly weak cosmology dependence, and we can therefore fit it using simulations from only one cosmology, saving a lot of computational time. Our function has been fitted using a large suite of available $N$-body simulation data and can easily be incorporated in a Boltzmann code like CAMB or CLASS to scale from a nonlinear $P(k)$ for $\Lambda$ CDM (created for example using an emulator like Euclidemulator) to a nonlinear $P(k)$ for the Hu-Sawicki model.

The requirements for the accuracy of the matter power spectrum are dictated by large upcoming surveys like LSST [2] and Euclid [1]. Estimates for how accurate the power spectrum needs to be to take full advantage of the statistical power of such surveys vary from $1 \%-2 \%$ [20] down as small as $0.5 \%$ [21] for scales $k \lesssim 10 h \mathrm{Mpc}^{-1}$. However, this is ignoring ${ }^{1}$ model uncertainties in the way baryonic feedback affects the matter power spectrum. Baryonic effects are expected (from simulations and observations) to be as large as $10-30 \%$ for scales $1 \lesssim k \lesssim 10 h \mathrm{Mpc}^{-1}$ $[23,24]$. The accuracy of the newly released Euclidemulator is quoted as being approximately $1 \%$ accurate for $k \lesssim$ $1 h \mathrm{Mpc}^{-1}$ and for redshifts $z \lesssim 3.5$. Based on these

\footnotetext{
${ }^{1}$ Also note that common $N$-body algorithms in state-of-the art codes disagree at the approximately $1 \%$ level already at $k=1 \mathrm{~h} \mathrm{Mpc}^{-1}$ and at the approximately $3 \%$ level at $k=$ $10 h \mathrm{Mpc}^{-1}$ [22].
}

considerations, our aim is to produce a fit that is approximately $1 \%$ accurate for scales $k \lesssim 1 h \mathrm{Mpc}^{-1}$ and less than 5\% accurate for scales $1<k<10 h \mathrm{Mpc}^{-1}$ and covering redshifts $z \lesssim 3.5$.

The setup of this paper is as follows. In Sec. II, we describe the simulations we have used; in Sec. III, we discuss the cosmology dependence of the enhancement $P_{f(R)} / P_{\Lambda \mathrm{CDM}}$; in Sec. IV, we describe the fitting function we have created together with some tests; in Sec. V, we show an application of the fitting formula by computing forecasts for how well galaxy clustering and weak lensing in a Euclid-like survey will be at constraining $f(R)$ gravity; and we conclude in Sec. VI.

\section{SIMULATIONS}

We take advantage of a large set of simulations to make the fit. ${ }^{2}$ For more about how the modified gravity simulations are performed, see, e.g., Ref. [25] and references within.

The main simulation suite we use is ELEPHANT [26] (WMAP9 cosmology), which has $N=1024^{3}$ particles, $L=1024 \mathrm{Mpc} / h, \Omega_{m}=0.281, \Omega_{b}=0.046, h=0.6974$, $n_{s}=0.971$, and $\sigma_{8}=0.820\left(A_{s}=2.3 \times 10^{-9}\right)$ with $\left|f_{R 0}\right|=$ $\left\{10^{-4}, 10^{-5}, 10^{-6}, 0\right\}$. These simulations were run with the ECOSMOG code [27].

For the same cosmology as above, we have also run extra simulations (also with the ECOSMOG code) using $N=256^{3}$ particles in a $L=200 \mathrm{Mpc} / h$ box with $\left|f_{R 0}\right|=\left\{10^{-5}, 5 \times 10^{-6}, 2 \times 10^{-5}, 5 \times 10^{-5}, 0\right\}$. This simulation suite contains simulations of $\Lambda \mathrm{CDM}$ and $f(R)$ gravity with $\left|f_{R 0}\right|=10^{-5}$ for $\sigma_{8}=0.88$ and 0.72 that allows us to test the $\sigma_{8}$ dependence of the modified gravity power-spectrum enhancement.

We also use, mainly for testing and validation, the DUSTGRAIN simulations suite [28] (Planck 2015 cosmology), which has $N=768^{3}$ particles, $L=750 \mathrm{Mpc} / h$, $\Omega_{m}=0.31345, \Omega_{b}=0.0481, h=0.6731, n_{s}=0.9658$, and $\sigma_{8}=0.842 \quad\left(A_{s}=2.199 \times 10^{-9}\right) \quad$ with $\quad\left|f_{R 0}\right|=$ $\left\{10^{-4}, 5 \times 10^{-5}, 10^{-5}, 0\right\}$. This simulation suite contains simulations of $\Lambda \mathrm{CDM}$ and $f(R)$ gravity with $\left|f_{R 0}\right|=10^{-5}$ and with $\Omega_{m}=0.2$ and 0.4 that allows us to test the $\Omega_{m}$ dependence on the power spectrum. All these simulations have the same value of $\sigma_{8}$ and were run with the MG-GADGET code [29].

All the $f(R)$ simulations mentioned above have corresponding $\Lambda \mathrm{CDM}$ simulations run with the same initial conditions that allow us to extract ratios $P_{f(R)} / P_{\Lambda \mathrm{CDM}}$ that (on the largest scales) are free of cosmic variance. For each simulation, we have about 30 redshifts between $z=0$ and $z=3$ that we use to compute the fit.

\footnotetext{
${ }^{2}$ All the power-spectrum data that we used are available at https://github.com/HAWinther/FofrFittingFunction.
} 
The power spectra used for the fitting functions have been estimated using POWMES [30] and (for DUSTGRAIN) by codes made by us. These codes have been tested and shown to give accurate results so we do not expect any bias due to different power-spectrum evaluation codes. What could give rise to a bias is that some of the data we use come from different $\mathrm{N}$-body codes, but as shown in Ref. [25], even though the actual power spectrum varies between different code types [22], the enhancement $P_{f(R)}(k) / P_{\Lambda \mathrm{CDM}}(k)$ (with both spectra computed by the same code) does not, and this is all that goes into our fit below.

The effect of the size of the box, mass resolution, and cosmic variance on the matter power spectrum was investigated in Ref. [31]. We find that the spectra we extract can be trusted down to scales of $k \sim 5-10 h \mathrm{Mpc}^{-1}$ depending on redshift.

\section{VARIATION OF ENHANCEMENT WITH COSMOLOGICAL PARAMETERS}

The fit for $P_{f(R)} / P_{\Lambda \mathrm{CDM}}$ we perform in this paper uses $\mathrm{N}$-body data from one single cosmology. The reason is that the cosmology dependence of this ratio is expected to be weak for almost all of the standard parameters with the possible exception of $\Omega_{m}$ and $\sigma_{8}$ as these correlate with the efficiency of screening and with the growth rate of the matter density perturbation. There is also one additional effect that is potentially significant, which is how degenerate the enhancement is with baryonic feedback (see, e.g., Refs. [29,32] for a discussion on the size of these effects compared to the modified gravity enhancement).

In this section, we will go through the different cosmological parameters and check how much the modified gravity enhancement changes. We will use linear perturbation theory, the halo model, fast approximative $N$-body simulations [33], and full $N$-body simulations (for the cases we have this available) to investigate this.

By the halo model, we mean the prediction of Ref. [34], which combines the modified linear power spectrum with a modified one-halo contribution and a quasilinear correction motivated by higher-order perturbation theory [35]. It incorporates the chameleon mechanism through an implementation of the thin-shell approximation in the spherical collapse model [36]. This generates a mass and environment dependent spherical collapse density, from which an environmentally averaged modified peak threshold is determined that is used to compute the $f(R)$ modification and chameleon screening effects on the halo mass function and concentration determining the one-halo contribution. A comparison to other modeling techniques of the modified nonlinear matter power spectrum in $f(R)$ gravity can be found in Ref. [37].

The approximate $N$-body simulations we use are the comoving lagrangian acceleration (COLA) implementation of $f(R)$ gravity. COLA simulations are $\mathcal{O}(100-1000)$ faster than high-resolution $N$-body simulations but can reproduce the enhancement of the power spectrum to percent-level accuracy down to fairly nonlinear scales $k \lesssim 1-5 h \mathrm{Mpc}^{-1}$.

\section{A. Massive neutrinos}

The effects of massive neutrinos are highly degenerate with a modified gravity signal since massive neutrinos decrease the growth of structure on small scales, while modifications of gravity usually enhances the growth. However, we do not expect a big change in the enhancement (i.e., for fixed cosmological parameters), and this is what we see in Fig. 1 for $N$-body simulations with $\left|f_{R 0}\right|=$ $10^{-4}$ [38]. The variation is seen to be at the subpercent level for $k \lesssim 1 h \mathrm{Mpc}^{-1}$ and approximately $4 \%$ around $k=$ $1 h \mathrm{Mpc}^{-1}$ for the large value $m_{\nu}=0.6 \mathrm{eV}$.

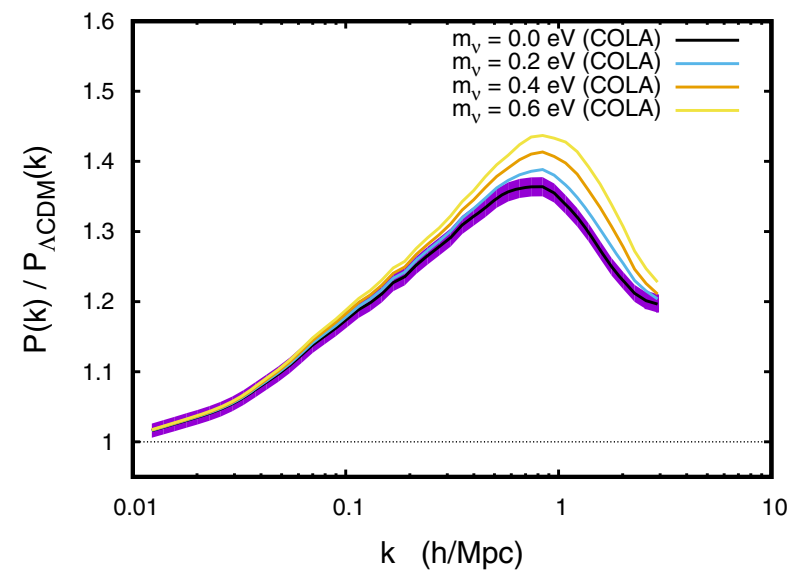

FIG. 1. Estimation for the variation of the boost factor $\frac{P(k)}{P_{\Lambda \mathrm{CDM}}(k)}$ with neutrino mass for $\left|f_{R 0}\right|=10^{-4}$ at $z=0$ based on $N$-body simulations (left) and COLA simulation (right). The shaded region corresponds to $\pm 1 \%$ of the central value. Note that the difference between the left and the right plot for $k \gtrsim 1 h \mathrm{Mpc}^{-1}$ comes from the fact that COLA simulations are not as accurate as high-resolution $N$-body simulations on very nonlinear scales. 

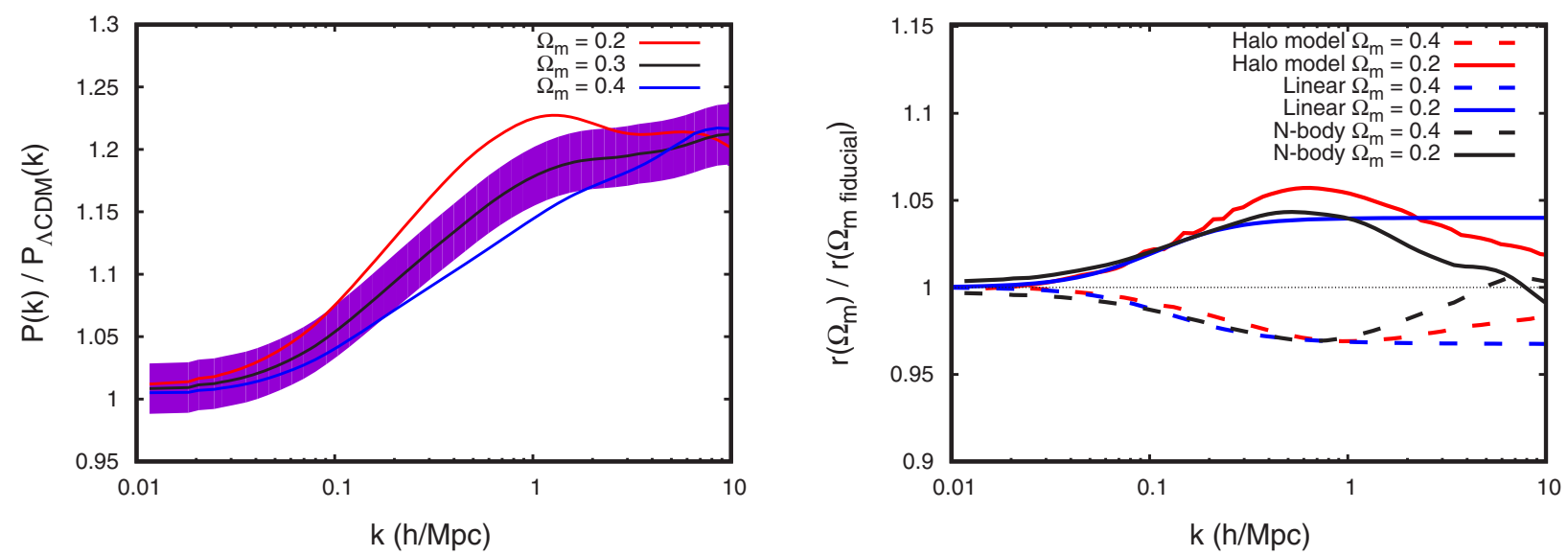

FIG. 2. Left: the enhancement $B=\frac{P(k)}{P_{\Lambda \mathrm{CDM}}(k)}$ for three different values of $\Omega_{m}$ for $\left|f_{R 0}\right|=10^{-5}$ at $z=0$ from $N$-body simulations. Right: variation of the enhancement $B\left(\Omega_{m}\right) / B\left(\Omega_{m}^{\text {fiducial }}\right)$ with respect to the fiducial value $\Omega_{m}^{\text {fiducial }}=0.3$ again for $\left|f_{R 0}\right|=10^{-5}$ at $z=0$. The shaded region corresponds to $\pm 2 \%$ of the central value.

\section{B. $\boldsymbol{\Omega}_{m}$}

Varying $\Omega_{m}$ changes the growth of linear perturbations, so it could potentially have a big effect on the enhancement. In Fig. 2, we show how much the enhancement changes for a fairly large variation of $\Omega_{m}$ (from $\Omega_{m}=0.3$ to $\Omega_{m}=0.2$ and $\Omega_{m}=0.4$ ) based on $N$-body simulations. This figure also shows that the correction is accurately captured by linear theory and/or the halo model.

A simple fit to results computed using linear theory at $z=0$ shows that the enhancement of the power spectrum $B_{\Omega_{m} \text { corr }} \equiv P(k) / P_{\Omega_{m}=0.3}(k)$ is given by

$$
B_{\Omega_{m} \text { corr }} \simeq 1-a \frac{\Delta \Omega_{m}}{\Omega_{m}} \tanh \left(\frac{k}{k_{*}}\right)^{b}
$$

with $\quad \frac{\Delta \Omega_{m}}{\Omega_{m}}=\frac{\Omega_{m}-0.3}{0.3}, \quad a=0.105, \quad b=1.4, \quad$ and $\quad k_{*}=$ $0.16\left(10^{-5} /\left|f_{R 0}\right|\right)^{1 / 2} h \mathrm{Mpc}^{-1}$ (in general, these parameters

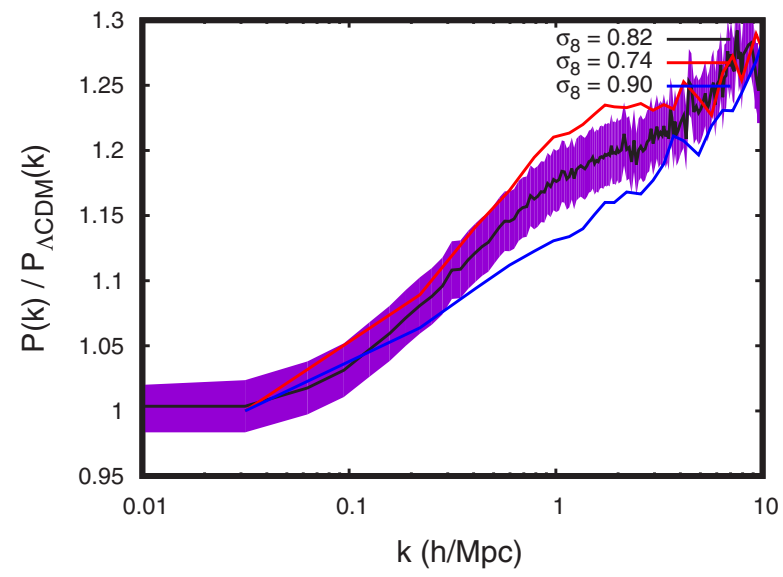

will depend on redshift). This gives us, for example, that a $10 \%$ change in $\Omega_{m}$ leads only to an approximately $1 \%$ change in the enhancement, so this is not a large effect, but it is straightforward to compute the correction using linear theory or the halo model if needed.

\section{Clustering amplitude}

In linear theory, there is no variation with $\sigma_{8}$ (or more technically speaking with $A_{s}$, the primordial amplitude). However, the amount of screening on nonlinear scales depends crucially on how clustered matter is, so this parameter could have a significant impact on nonlinear scales. Even in $\Lambda \mathrm{CDM}$, an enhancement of $\sigma_{8}$ leads to a greater enhancement of the clustering on nonlinear scales than as predicted by linear theory. In Fig. 3, we show how large this variation is based on $N$-body simulations together with predictions from both linear theory and the halo model.

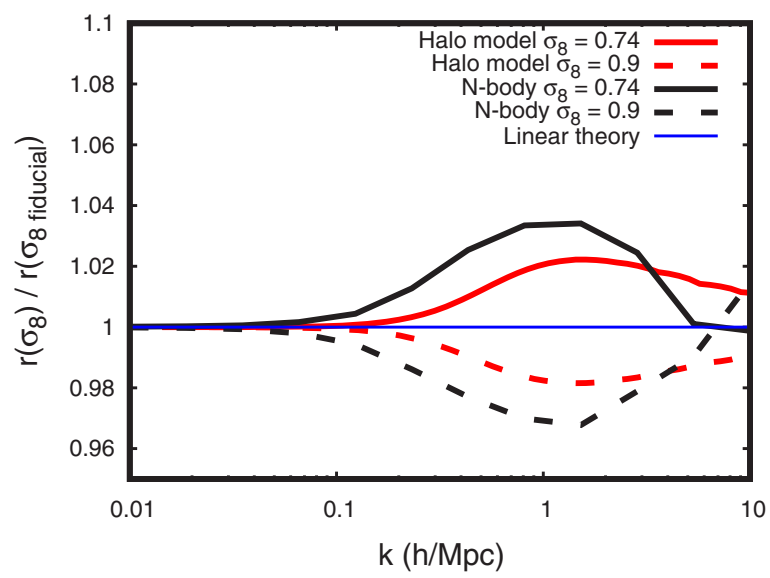

FIG. 3. Left: the enhancement $B=\frac{P(k)}{P_{\Lambda \mathrm{CDM}}(k)}$ for three different values of $\sigma_{8}$ for $\left|f_{R 0}\right|=10^{-5}$ at $z=0$ from $N$-body simulations. Right: variation of the enhancement $r\left(\sigma_{8}\right) / r\left(\sigma_{8}^{\text {fiducial }}\right)$ with respect to the fiducial value $\sigma_{8}^{\text {fiducial }}=0.82$ again for $\left|f_{R 0}\right|=10^{-5}$ at $z=0$. The shaded region corresponds to $\pm 2 \%$ of the central value. 
TABLE I. The best-fit parameters for the enhancement of the linear power spectrum for $\left|f_{R 0}^{\text {fid }}\right|=10^{-5}$.

\begin{tabular}{llcc}
\hline \hline & $i=0$ & $i=1$ & $i=2$ \\
\hline$b_{0 i}$ & 3.10 & 2.34466 & -1.86362 \\
$c_{0 i}$ & 34.4951 & 28.8637 & -13.1302 \\
$d_{0 i}$ & 0.14654 & -0.0100 & -0.14944 \\
$e_{0 i}$ & 1.62807 & 0.71291 & -1.41003 \\
\hline \hline
\end{tabular}

A fit to our simulations with varying $\sigma_{8}(0.72,0.8$, and 0.88 ) shows that the effect of varying $\sigma_{8}$ can be described by a multiplicative correction to the enhancement which for $\left|f_{R 0}\right|=10^{-5}$ at $z=0$ and for $k \lesssim 10 h \mathrm{Mpc}^{-1}$ is approximately given by

$$
B_{\sigma_{8} \text { corr }} \simeq 1+\frac{\Delta \sigma_{8}}{\sigma_{8}} \frac{k}{\left(1+\left(k / k_{*}\right)\right)^{2}},
$$

where $k_{*}=1.2 \mathrm{~h} \mathrm{Mpc}^{-1}$ and $\frac{\Delta \sigma_{8}}{\sigma_{8}} \equiv \frac{\sigma_{8}-0.8}{0.8}$. A $10 \%$ deviation of $\sigma_{8}$ from its fiducial value ( 0.8$)$ leads to an approximately $2 \%-3 \%$ level deviation in the enhancement for medium wave numbers.

\section{Other parameters}

Linear perturbation theory predicts zero variation with other cosmological parameters such as the Hubble constant $H_{0}$ (when the fitting function is expressed in terms of $k$ in units of $h \mathrm{Mpc}^{-1}$ ) and the spectral index $n_{s}$. Changing the spectral index does modify the amplitude of clustering on small scales and could also influence screening; however, we have checked using the halo model and COLA simulations that the expected variation is much less than $1 \%$ for all scales of interest within a reasonable variation in these parameters (here defined to be $3 \sigma$ of the Planck 2018 cosmological constraints).

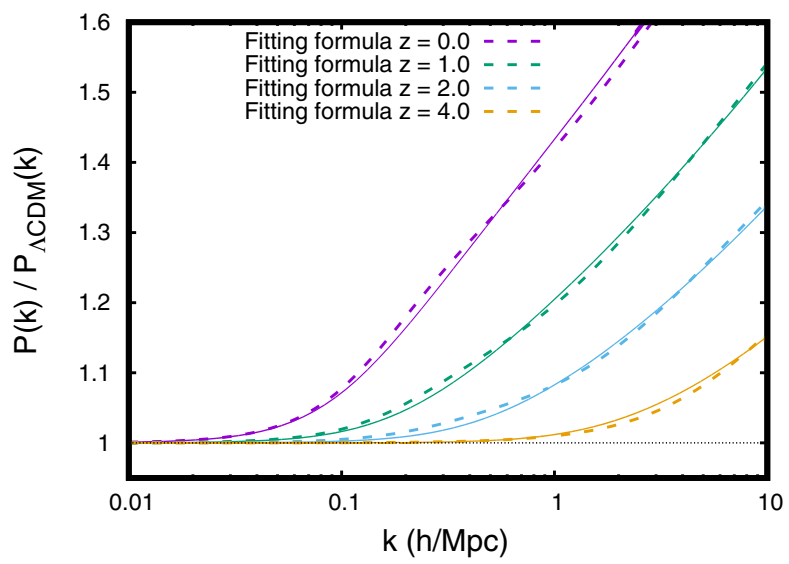

FIG. 4. Comparison of the fitting formula for the linear enhancement to the exact result from linear perturbation theory (here for $\left|f_{R 0}\right|=10^{-5}$ ).
TABLE II. The best-fit values using the data from $\left|f_{R 0}\right|=$ $\left\{10^{-5}, 5 \times 10^{-6}, 10^{-6}, 10^{-7}\right\}$ with $\left|f_{R 0}\right|^{\text {fid }}=5 \times 10^{-6}$ (left), from $\left|f_{R 0}\right|=\left\{5 \times 10^{-5}, 10^{-5}, 5 \times 10^{-6}\right\}$ with $\left|f_{R 0}\right|$ fid $=10^{-5}$ (middle), and from $\left|f_{R 0}\right|=\left\{10^{-4}, 5 \times 10^{-5}, 10^{-5}\right\} \quad$ with $\left|f_{R 0}\right|^{\text {fid }}=5 \times 10^{-5}$ (right).

\begin{tabular}{|c|c|c|c|}
\hline & $i=0$ & $i=1$ & $i=2$ \\
\hline$b_{0 i}$ & 0.76878 & 0.22638 & 0.00759 \\
\hline$b_{1 i}$ & -0.40537 & -0.10711 & -0.00102 \\
\hline$b_{2 i}$ & 0.00752 & 0.04846 & 0.01180 \\
\hline$c_{0 i}$ & 0.02886 & -0.02438 & -0.02963 \\
\hline$c_{1 i}$ & -0.06382 & -0.05196 & 0.02597 \\
\hline$c_{2 i}$ & -0.40121 & -0.03518 & 0.07688 \\
\hline$d_{0 i}$ & 1.00000 & 0.10901 & 0.12027 \\
\hline$d_{1 i}$ & 0.00000 & 0.08189 & 0.02492 \\
\hline$d_{2 i}$ & 0.00000 & -0.05682 & -0.02985 \\
\hline$e_{0 i}$ & 0.36951 & 0.14719 & 0.03127 \\
\hline$e_{1 i}$ & 0.10939 & 0.06176 & 0.02933 \\
\hline$e_{2 i}$ & -0.34209 & -0.13138 & -0.01419 \\
\hline$f_{0 i}$ & 1.03544 & -0.13912 & -0.05656 \\
\hline$f_{1 i}$ & -0.26277 & -0.13231 & -0.03389 \\
\hline$f_{2 i}$ & 0.23028 & 0.13132 & 0.07127 \\
\hline$g_{0 i}$ & 0.20246 & 0.06323 & 0.05229 \\
\hline$g_{1 i}$ & -0.11611 & 0.06943 & 0.07807 \\
\hline$g_{2 i}$ & 0.10245 & 0.00296 & -0.09770 \\
\hline$b_{0 i}$ & 0.93650 & -0.03999 & 0.24007 \\
\hline$b_{1 i}$ & -0.54583 & 0.30370 & 0.18820 \\
\hline$b_{2 i}$ & 0.63480 & 0.36096 & 0.66583 \\
\hline$c_{0 i}$ & -0.02906 & 0.00062 & 0.01222 \\
\hline$c_{1 i}$ & -0.09544 & -0.00942 & -0.03434 \\
\hline$c_{2 i}$ & -0.34249 & -0.01813 & -0.05204 \\
\hline$d_{0 i}$ & 1.00000 & 0.39355 & 0.77661 \\
\hline$d_{1 i}$ & 0.00000 & 0.29088 & 0.47078 \\
\hline$d_{2 i}$ & 0.00000 & -0.41149 & -0.68192 \\
\hline$e_{0 i}$ & 0.49107 & 0.37630 & 0.26101 \\
\hline$e_{1 i}$ & 0.29782 & 0.48636 & 0.52563 \\
\hline$e_{2 i}$ & -0.28714 & 0.03494 & 0.26626 \\
\hline$f_{0 i}$ & 0.92041 & -0.09308 & 0.27038 \\
\hline$f_{1 i}$ & -0.28239 & 0.07838 & 0.37029 \\
\hline$f_{2 i}$ & 0.53954 & 0.19496 & 0.19486 \\
\hline$g_{0 i}$ & 0.31864 & 0.03340 & -0.00276 \\
\hline$g_{1 i}$ & 0.04570 & 0.07630 & 0.04616 \\
\hline$g_{2 i}$ & 0.13924 & -0.00010 & 0.18990 \\
\hline$b_{0 i}$ & 0.57248 & 0.49880 & 0.57426 \\
\hline$b_{1 i}$ & 0.25469 & 0.36089 & -0.30799 \\
\hline$b_{2 i}$ & 1.21637 & 0.07034 & 0.83164 \\
\hline$c_{0 i}$ & 0.00046 & 0.02574 & -0.00936 \\
\hline$c_{1 i}$ & -0.09012 & 0.01689 & 0.00221 \\
\hline$c_{2 i}$ & -0.35585 & -0.03070 & 0.00768 \\
\hline$d_{0 i}$ & 1.00000 & 0.77903 & 1.26756 \\
\hline$d_{1 i}$ & 0.00000 & 0.91964 & 1.44477 \\
\hline$d_{2 i}$ & 0.00000 & -0.93633 & -1.44129 \\
\hline$e_{0 i}$ & 2.31154 & -0.20699 & -0.65038 \\
\hline$e_{1 i}$ & 2.29822 & 0.26608 & 0.01792 \\
\hline$e_{2 i}$ & -0.48319 & 0.60336 & 0.92704 \\
\hline$f_{0 i}$ & 1.21959 & -0.25171 & -0.14644 \\
\hline$f_{1 i}$ & 0.35388 & 0.12487 & 0.02003 \\
\hline$f_{2 i}$ & 1.02533 & 0.34599 & 0.08923 \\
\hline$g_{0 i}$ & 0.28475 & -0.04719 & 0.09592 \\
\hline$g_{1 i}$ & -0.15829 & 0.13977 & 0.36819 \\
\hline$g_{2 i}$ & 0.54118 & -0.13489 & -0.15783 \\
\hline
\end{tabular}



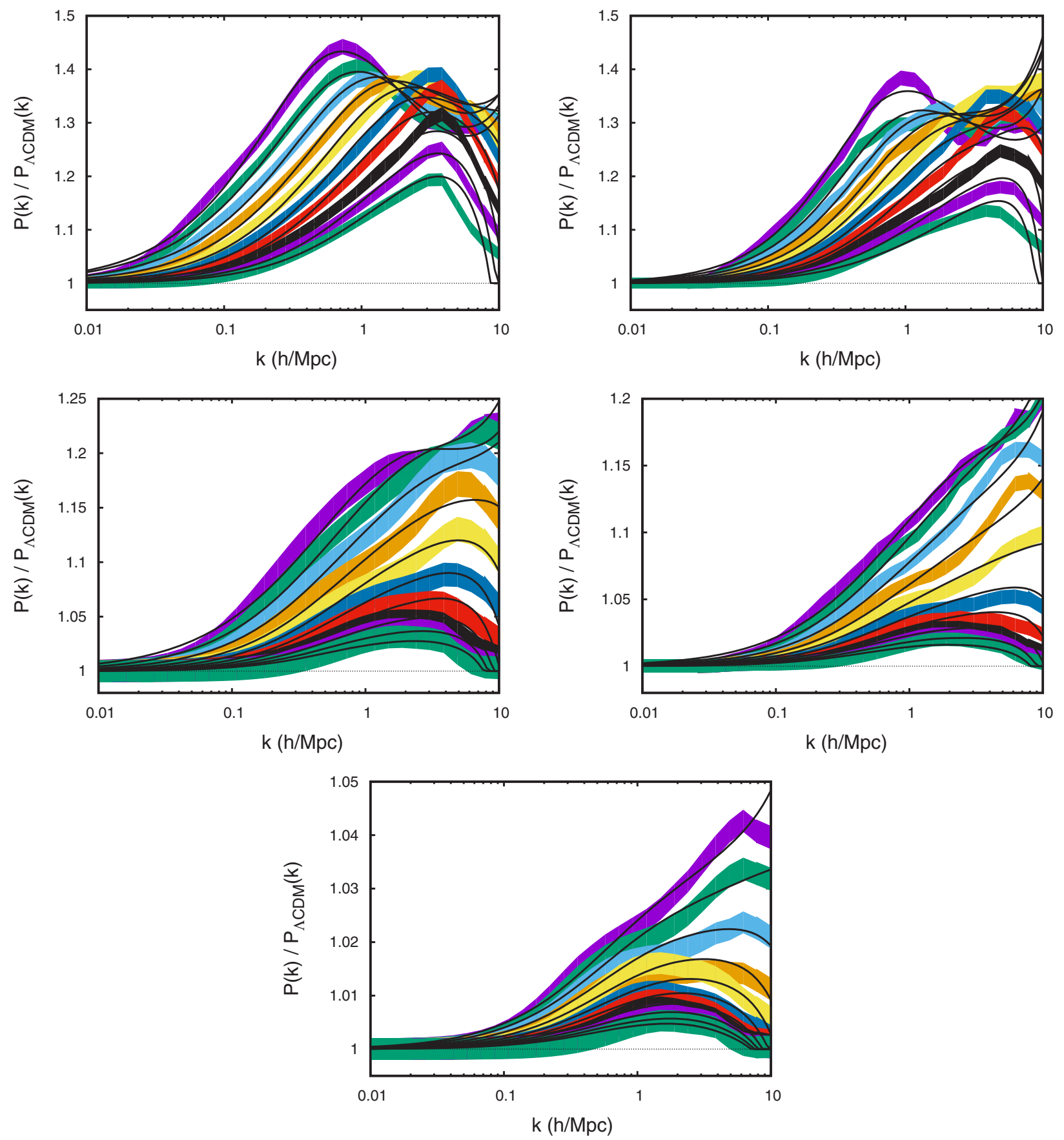

FIG. 5. Fitting function compared to $N$-body data for $\left|f_{R 0}\right|=10^{-4}$ (up left), $\left|f_{R 0}\right|=5 \times 10^{-5}$ (up right), $\left|f_{R 0}\right|=10^{-5}$ (middle left), $\left|f_{R 0}\right|=5 \times 10^{-6}$ (middle right), and $\left|f_{R 0}\right|=10^{-6}$ (bottom) and $z=\{0.0,0.259,0.518,0.777,1.036,1.294,1.554,1.812,2.071,2.330\}$. The shaded region is to give the reader an idea of how good the fitting function is and denotes $\pm 1 \%$ for all plots except for $\left|f_{R 0}\right|=5 \times 10^{-6}$ and $\left|f_{R 0}\right|=10^{-6}$ where it is $\pm 0.5 \%$ and $\pm 0.2 \%$ respectively.

\section{FITTING FUNCTIONS}

\section{A. Fitting function for the linear enhancement}

At the level of linear perturbations, the growth of matter perturbations is determined by

$$
\ddot{\delta}+2 H \dot{\delta}=\frac{3}{2} \Omega_{m}(a) H^{2} \delta\left(1+\frac{1}{3} \frac{k^{2}}{k^{2}+m^{2} a^{2}}\right),
$$

where

$$
m^{2}(a)=\frac{H_{0}^{2}\left(\Omega_{m}+4 \Omega_{\Lambda}\right)}{2\left|f_{R 0}\right|}\left(\frac{\Omega_{m} a^{-3}+4 \Omega_{\Lambda}}{\Omega_{m}+4 \Omega_{\Lambda}}\right)^{3},
$$

from which it follows that the enhancement of the linear matter power spectrum for a general model is simply the enhancement of a fiducial model evaluated at 


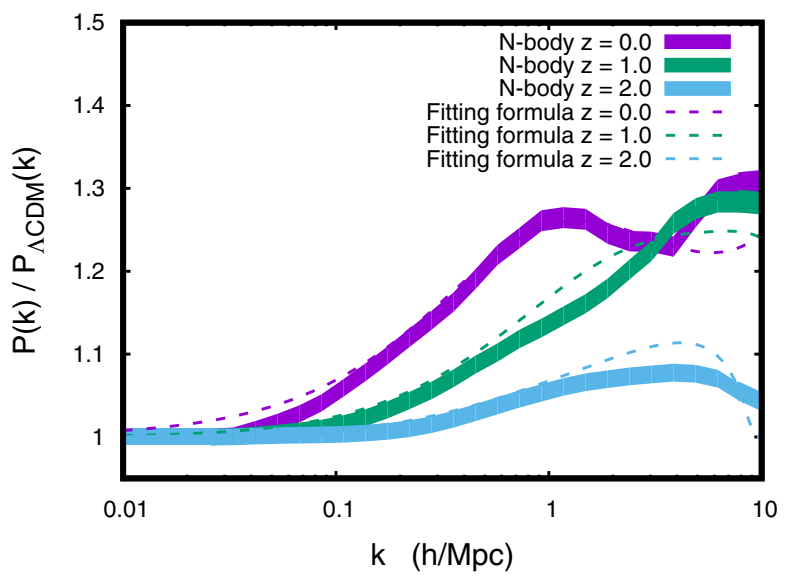

FIG. 6. Test of the fitting function to $N$-body data not used in the fit (left), i.e., simulations with $\left|f_{R 0}\right|=2 \times 10^{-5}$. The shaded region corresponds to $\pm 1 \%$ of the central value.

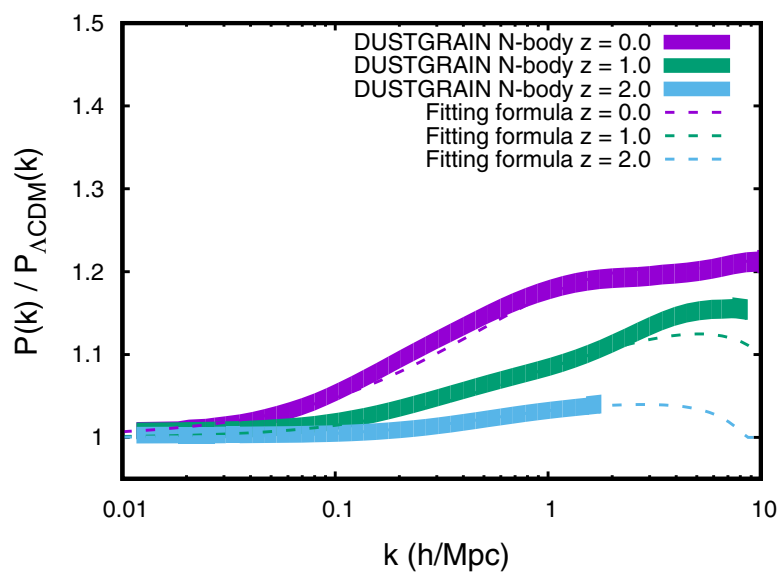

FIG. 7. Test of the fitting function to $N$-body data with a different background cosmology (DUSTGRAIN vs ELEPHANT) for $\left|f_{R 0}\right|=10^{-5}$. The shaded region corresponds to $\pm 1 \%$ of the central value.

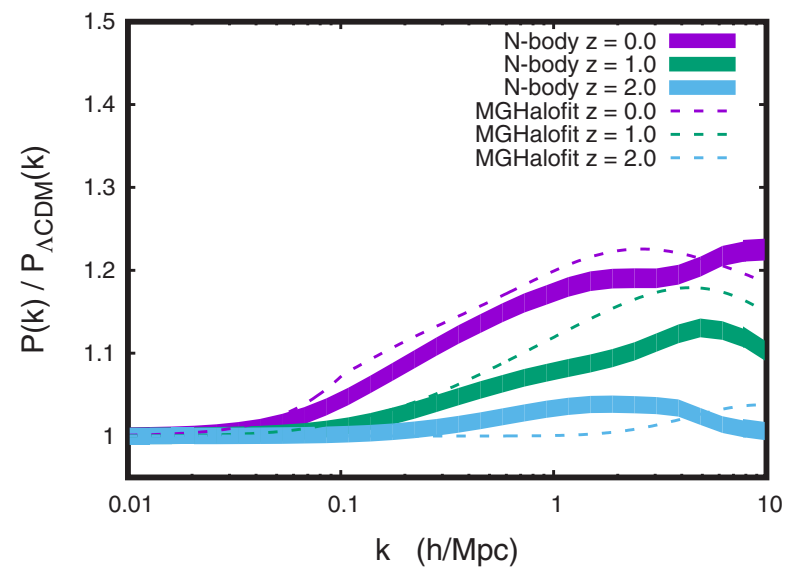

$k^{*}=k\left(f_{R 0} / f_{R 0}^{\mathrm{fid}}\right)^{1 / 2}$. We perform a fit using the fitting function

$$
\begin{aligned}
\frac{P_{\Lambda}^{\text {linear fid }}(k, z)}{P_{\Lambda}^{\text {linear }} \operatorname{CDM}(k, z)}= & 1+\frac{(b(a) k)^{2}}{1+c(a) k^{2}} \\
& +d(a)\left|\frac{\log (k) k}{k-1}\right| \arctan (e(a) k),
\end{aligned}
$$

which is constructed to interpolate between the expected low and high $k$ limits [approximately $1+b k^{2}$ for small $k$ and approximately $\log (k)$ for large $k]$. Similar functional forms for the fitting functions of the enhancement within a coupled dark energy model were used in Ref. [18]. The functions, $X=b, c, d, e$, above are written as

$X\left(z, f_{R 0}\right)=X_{0}(r)+X_{1}(r)(a-1)+X_{2}(r)(a-1)^{2}$,

where $r=\log \left(f_{R 0} / f_{R 0}^{\mathrm{fid}}\right)$, leaving us with 12 free parameters to fit. The best fit from taking $\left|f_{R 0}\right|$ fid $=10^{-5}$ using $\Omega_{m}=0.281$ is shown in Table I, and in Fig. 4, we show a comparison to the true result.

\section{B. Fitting function for the nonlinear enhancement}

The fitting function we use for our fit is given by

$$
\begin{aligned}
\frac{P_{f(R)}(k, z)}{P_{\Lambda \mathrm{CDM}}(k, z)}= & 1+b\left(z, f_{R 0}\right) \frac{\left(1+c\left(z, f_{R 0}\right) \cdot k\right)}{\left(1+d\left(z, f_{R 0}\right) \cdot k\right)} \\
& \left.\times \arctan \left(e\left(z, f_{R 0}\right) \cdot k\right)\right)^{f\left(z, f_{R 0}\right)+g\left(z, f_{R 0}\right) \cdot k},
\end{aligned}
$$

where (for $X=b, c, d, e, f, g$ )

$$
X\left(z, f_{R 0}\right)=X_{0}(r)+X_{1}(r)(a-1)+X_{2}(r)(a-1)^{2}
$$

with

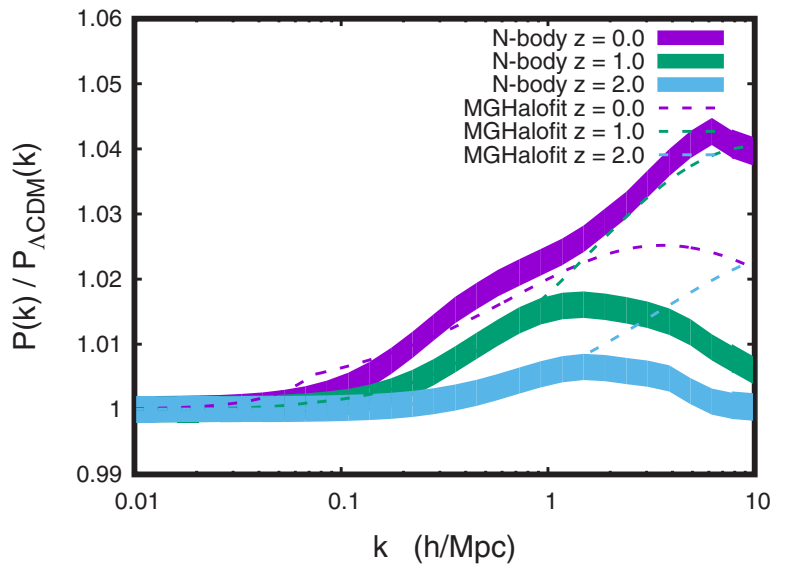

FIG. 8. Comparison of MGHALOFIT to our $N$-body data for $\left|f_{R 0}\right|=10^{-5}$ (left) and $10^{-6}$ (right). The shaded region corresponds to $\pm 1 \%$ (left) and $\pm 0.2 \%$ (right) of the central value. 

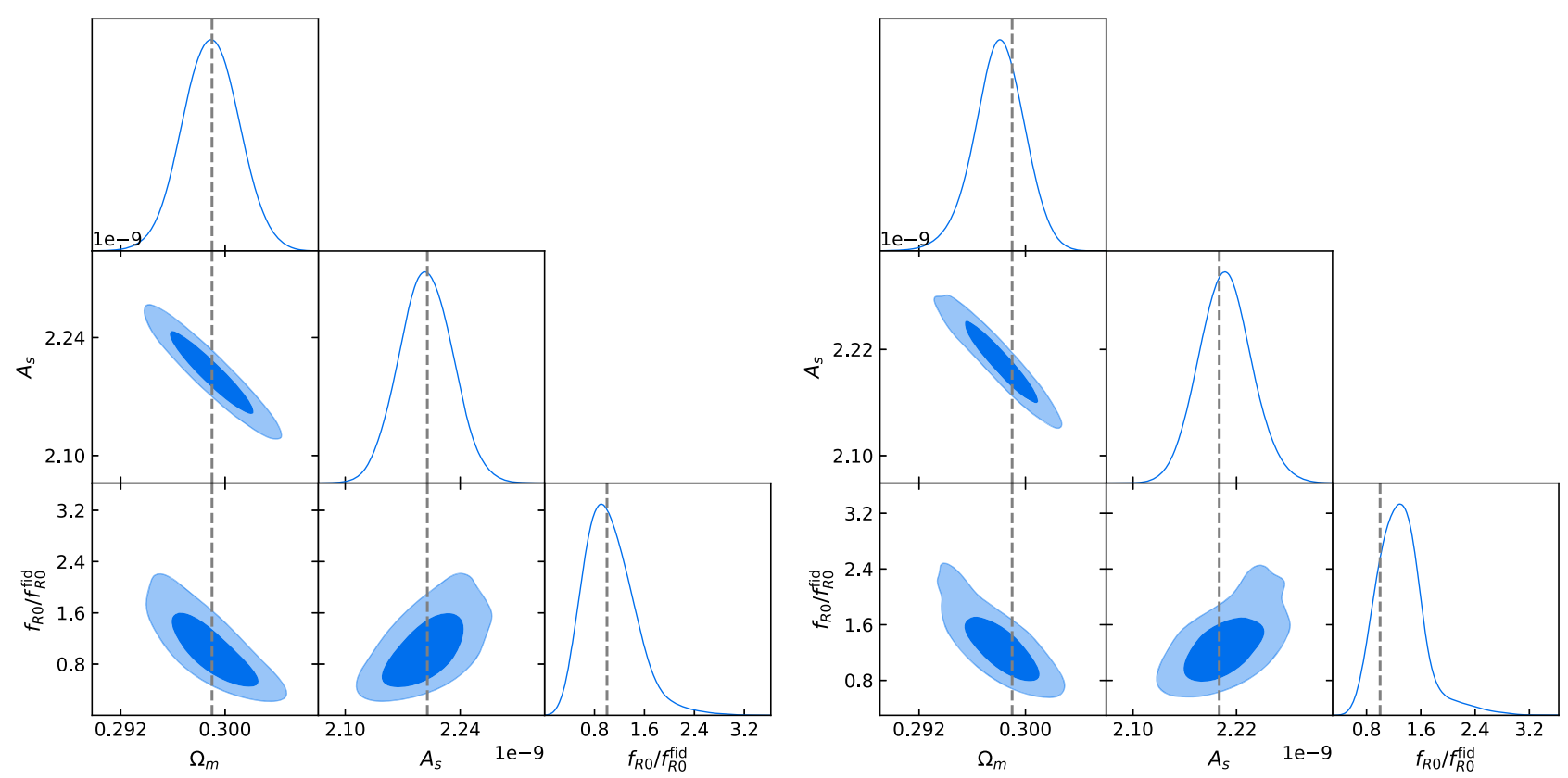

FIG. 9. Test of the fitting function by fitting to mock data, using the $N$-body enhancement for $\left|f_{R 0}\right|=2 \times 10^{-5}$ around $z=1$. We show the fits for the two values $k_{\max }=1 \mathrm{~h} \mathrm{Mpc}^{-1}$ (left) and $k_{\max }=5 \mathrm{~h} \mathrm{Mpc}^{-1}$ (right).

$$
X_{i}(r)=X_{i 0}+X_{i 1} r+X_{i 2} r^{2}
$$

where $r=\log \left(f_{R 0} / f_{R 0}^{\text {fid }}\right)$. This makes 54 free parameters for the full $f_{R 0}$, scale, and redshift dependence.

We choose to make three different fits, one using $\left|f_{R 0}\right|=\left\{10^{-4}, 5 \times 10^{-5}, 10^{-5}\right\}$ (high), one using $\left|f_{R 0}\right|=$ $\left\{10^{-5}, 5 \times 10^{-6}, 10^{-6}\right\}$ (medium), and one using $\left|f_{R 0}\right|=$ $\left\{10^{-5}, 5 \times 10^{-6}, 10^{-6}, 10^{-7}\right\}$ (low), and then interpolate between these (overlapping) fits. The best-fit parameters we find are given in Table II, and the agreement as a function of scale and redshift can be seen in Fig. 5. This figure shows that the fit has percent-level accuracy for most scales and redshifts, with the exception of the smallest scales for the largest values of $\left|f_{R 0}\right|$.

In Fig. 6, we perform a test of our fitting function by predicting the enhancement for $\left|f_{R 0}\right|=2 \times 10^{-5}$, a value that was not used to generate the fit. This value is in the middle of $\left|f_{R 0}\right|=10^{-5}$ and $\left|f_{R 0}\right|=5 \times 10^{-5}$ and should therefore give us a good estimate for the accuracy of our fit. The agreement is within approximately $1 \%-2 \%$ for $k \lesssim$ $1 \mathrm{~h} \mathrm{Mpc}-1$ and approximately $2 \%-3 \%$ for $k \lesssim 10 h \mathrm{Mpc}^{-1}$.

In Fig. 7, we test our fitting function by comparing it to simulations with a different background cosmology from that which is used to make the fit. As expected from the discussion in the previous section, the agreement is very good.

These numbers should be contrasted to the enhancement of the matter power spectrum itself relative to $\Lambda \mathrm{CDM}$, which is typically $10 \%-40 \%$.

A fitting function, based on HALOFIT, for the Hu-Sawicki model already exists in the literature, namely MGHALOFIT.
In Fig. 8, we show a comparison of our fitting formula to MGHALOFIT, which shows that our fit performs better.

To test the fitting function, we create mock data and try to perform a fit to $P(k, z)$ for a Euclid-like survey with $V=50(\mathrm{Gpc} / h)^{3}$ with $n_{\text {gal }}=10^{-3}(\mathrm{Mpc} / h)^{-3}$ using the diagonal likelihood

$$
\begin{aligned}
\log \mathcal{L}= & -\frac{1}{2} \sum_{k, z}\left(P(k, z)-P^{\mathrm{fid}}(k, z)\right) \\
& \times C^{-1}\left(P(k, z)-P^{\mathrm{fid}}(k, z)\right)
\end{aligned}
$$

where $C^{-1}=\frac{V k^{2} \Delta k}{4 \pi^{2}\left(P^{+\mathrm{fid}}(k, z)+1 / n\right)^{2}}$. The sum is over six evenly spaced $z$ bins from $z=0$ to $z=2$ and 30 logarithmically spaced $k$ bins between $k=10^{-4} h \mathrm{Mpc}^{-1}$ and $k=$ $5 h \mathrm{Mpc}^{-1}$. This assumes Gaussian fluctuations on all scales which significantly underestimate the errors on nonlinear scales and also does not take into account uncertainty of unknown baryonic physics. Thus, any fit based on this would be completely dominated by the smallest scales. To get more realistic errors, we try to take this into account by imposing a minimum $1 \%$ error on nonlinear scales starting at $k=0.5 \mathrm{~h} \mathrm{Mpc}^{-1}$ and growing to $10 \%$ at $k=10 \mathrm{~h} \mathrm{Mpc}^{-1}$. The fiducial power spectrum is generated using the Eisenstein-Hu fitting function for $\Lambda$ CDM $[39,40]$, converted to a nonlinear power spectrum using HALOFIT and finally multiplied by the $f(R)$ enhancement found in simulations with $\left|f_{R 0}\right|=2 \times 10^{-5}$. The result of fits to $P(k, z)$ around $z=1$ (where our fit is seen to deviate a bit from the $N$-body result) can be found in Fig. 9. We also performed this test for other redshifts with similar results. 

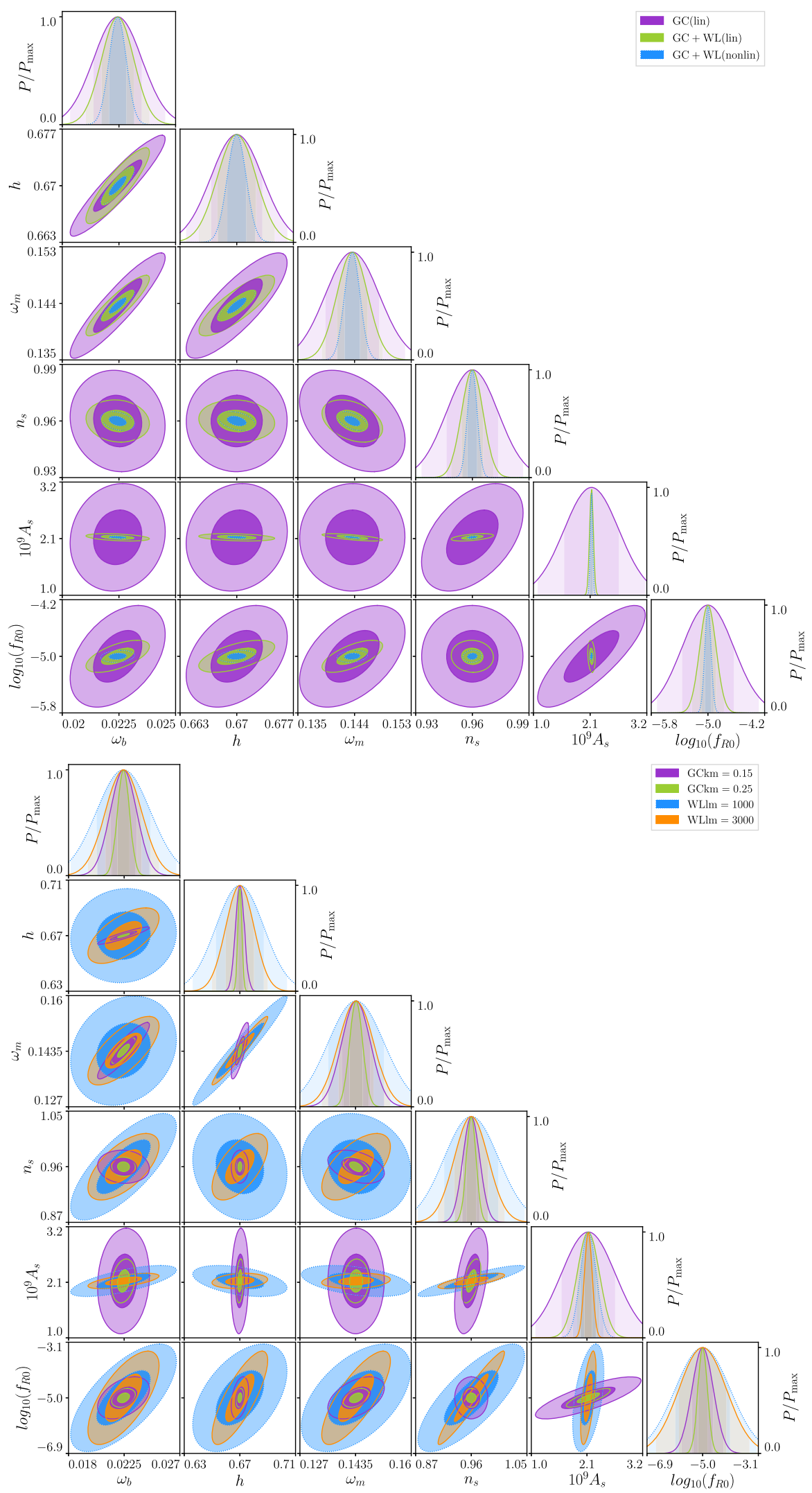

FIG. 10. Forecasted constraints on the Hu-Sawicki model, for the fiducial value $\left|f_{R 0}\right|=10^{-5}$, from GC and WL in a Euclid-like survey for different values of $k_{\max }$ and $\ell_{\max }$. In the figure, (lin) refers to $k_{\max }=0.15 \mathrm{~h} \mathrm{Mpc}^{-1}, \ell_{\max }=1000$, and (nonlin) refers to $k_{\max }=0.25 h \mathrm{Mpc}^{-1}, \ell_{\max }=3000$. 
TABLE III. Constraints on the parameters in the Hu-Sawicki model, for the fiducial value $\left|f_{R 0}\right|=10^{-5}$, coming from galaxy clustering (above), weak lensing (middle), and combined (below) for two different values of $k_{\max }$ and $\ell_{\max }$.

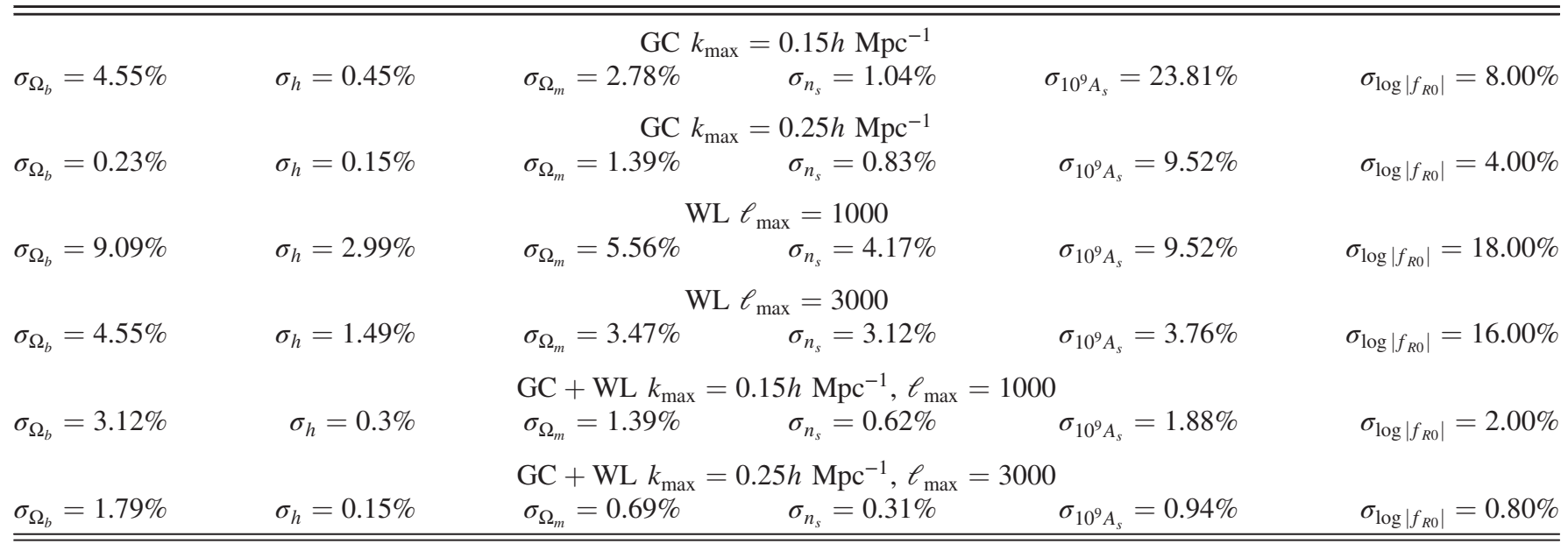

\section{FORECAST}

In this section, we will give an example application of the fitting formula presented in this paper by using it to compute forecasts for how well the Hu-Sawicki model can be constrained in future surveys (see Ref. [41] for forecasts for general scalar-tensor theories). We adopt the Fisher-matrix formalism to the main cosmological observables for next-generation galaxy surveys, namely galaxy clustering (GC) and weak lensing (WL). The matter power spectrum is computed using CAMB with the HALOFIT prescription to get the nonlinear power spectrum for $\Lambda \mathrm{CDM}$ and then use our fitting formula to go from $\Lambda \mathrm{CDM}$ to $f(R)$. We neglect cross-correlations between GC and WL. To perform our forecasts, we use the survey parameters for a Euclid-like mission [42].

For GC, the main observable is the observed (redshiftspace) galaxy power spectrum, which we model as

$$
\begin{aligned}
P_{g}(k, \mu, z)= & \frac{D_{A, f}^{2}(z) / H_{f}(z)}{D_{A}^{2}(z) / H(z)}\left(b(z)+f(z) \mu^{2}\right)^{2} \\
& \times e^{-k^{2} \mu^{2} \sigma^{2}} P_{\mathrm{DM}}(k, z),
\end{aligned}
$$

where $D_{A}$ is the angular diameter distance, $H$ is the Hubble function, $\mu$ is the cosine of the angle between the line of sight and the Fourier vector $\vec{k}, f(z)$ is the growth function, and $\sigma^{2} \equiv \sigma_{r}^{2}+\sigma_{v}^{2}$ is a parameter parametrizing errors induced by spectroscopic redshift measurements $\left(\sigma_{r}\right)$ and the fingers-of-god effect $\left(\sigma_{v}\right)$ that we marginalize over. A subscript ${ }_{f}$ denotes the value in the fiducial cosmology, which we take to be a $\Lambda \mathrm{CDM}$ cosmology with parameters $n_{s}=0.96,10^{9} A_{s}=2.126, h=0.67, \Omega_{m}=0.32, \Omega_{b}=$ $0.05, \Omega_{\Lambda}=0.68, m_{\nu}=0.06 \mathrm{eV}$, and $\sigma_{v}=300 \mathrm{~km} / \mathrm{s}$. The fiducial $f(R)$ parameter is taken to be $\left|f_{R 0}\right|=10^{-5}$.
The Fisher matrix for GC is taken to be

$$
F_{i j}=\frac{V_{\text {survey }}}{8 \pi^{2}} \int_{-1}^{1} \mathrm{~d} \mu \int_{k_{\min }}^{k_{\max }} \mathrm{d} k\left[\frac{\partial D}{\partial \theta_{i}} D^{-1} \frac{\partial D}{\partial \theta_{j}} D^{-1}\right],
$$

where the data vector $D=P_{g}(k, \mu, z)+1 / n(z)$ with $V_{\text {survey }}$ being the survey volume and $n(z)$ being the galaxy number density. We compute the constraints for two different values of $k_{\max }=0.15$ and 0.25 both with $k_{\min }=0.008 \mathrm{~h} / \mathrm{Mpc}$. For computing the Fisher matrix, we used $9 z$ bins in the range $z=0.95$ and $z=1.75$.

The second probe we include is WL cosmic shear: the distortions in the ellipticities of galaxies due to bending of light around large cosmic structures. The cosmic shear is computed using ten redshift bins in redshift range $0<z<2.5$. The cosmic shear at a redshift bin $i$ is correlated with the cosmic shear at another redshift bin $j$ since light coming from each bin will propagate through some of the same structures on the way to us. The cross-power spectrum of cosmic shear in bins $i$ and $j$ is determined by the underlying dark matter power spectrum via

$$
\begin{aligned}
C_{i j}(\ell)= & \frac{9}{4} \int_{0}^{\infty} \mathrm{d} z \frac{W_{i}(z) W_{j}(z) H^{3}(z) \Omega_{m}^{2}(z)}{(1+z)^{4}} \\
& \times P_{\mathrm{DM}}(k=\ell / r(z), z)
\end{aligned}
$$

where $r(z)$ is the comoving distance and $W$ is a a window function given by the photometric redshift distribution function and the galaxy number density distribution.

The Fisher matrix for WL is given by

$$
F_{i j}=f_{\text {sky }} \sum_{\ell}^{\ell_{\max }} \frac{(2 \ell+1) \Delta \ell}{2} \operatorname{tr}\left[\frac{\partial C}{\partial \theta_{i}} \operatorname{Cov}^{-1} \frac{\partial C}{\partial \theta_{j}} \operatorname{Cov}^{-1}\right],
$$


where $C$ is a matrix with elements $C_{i j}, f_{\text {sky }}=0.36$ $\left(15000 \mathrm{deg}^{2}\right)$ is the sky fraction covered by the survey, $\Delta \ell$ is the size of the $\ell$ bins, $\ell_{\max }$ is the maximum multipole number, and $\mathrm{Cov}$ is the WL covariance matrix. In this paper, we consider the two values $\ell_{\max }=1000$ and 3000 . We used $100 \operatorname{logarithmically~spaced~} \ell$ bins between $\ell_{\min }=$ 100 and $\ell_{\max }$, and we used ten evenly spaced $z$ bins between $z=0.15$ and $z=2.5$. Apart from the particular numbers quoted above, we use the same setup as in Ref. [43], so see that paper for more details.

In Fig. 10, we show the Fisher forecast constraints we obtained using the fiducial value $\left|f_{R 0}\right|=10^{-5}$, which is slightly below the best constraints from current cosmological data (see, e.g., Ref. [44]). We show how the results change when going from only using fairly linear scales to including more and more nonlinear scales $\left(k_{\max }=\right.$ $0.15 \mathrm{hMpc}^{-1}, \ell_{\max }=1000$ vs $k_{\max }=0.25 \mathrm{~h} \mathrm{Mpc}^{-1}, \ell_{\max }=$ 3000 ) in the forecast. In Table III, we show the marginalized constraints on the cosmological parameters for the different cases we have considered.

GC and WL are individually able to constrain $\log _{10}\left|f_{R 0}\right|$ to approximately 5\% and $15 \%$ respectively depending on how many nonlinear modes we include in the forecast. The modified gravity parameter $f_{R 0}$ is seen to be mostly degenerate with the clustering amplitude $A_{s}$. Combining GC and WL, we are able to break this degeneracy and bring the potential constraints down to approximately 1-2\% $\left(\Delta f_{R 0} \lesssim 2 \times 10^{-6}\right)$. However, we caution that this is a simplified forecast not taking baryonic effects on the matter power spectrum or including massive neutrinos, which both are known to be degenerate with a potential modified gravity signal $[38,45]$.

\section{CONCLUSIONS}

In this paper, we have presented a fitting function for the linear and nonlinear matter power spectrum of the Hu-Sawicki $f(R)$ model using power spectra computed from $N$-body simulations for several different values of the model parameters. This is one of the most studied modified gravity models and often the fiducial choice when trying to constrain modified gravity effects in observational data.

We have shown that the enhancement has a weak cosmology dependence, which allows us to make the fit for a fixed cosmology. Any cosmology dependence can be accurately included by using inexpensive tools like linear theory, the halo model, or COLA simulations.

We have demonstrated that the fitting function is accurate over a large range of scales and redshifts. We also tested it against the existing MGHALOFIT code and found that our fitting function generally performs better.

One can easily integrate our fitting function in any approach that produces a nonlinear matter power spectrum for $\Lambda \mathrm{CDM}$. With this paper, we provide the fitting function already implemented ${ }^{3}$ in many common programming languages like C, Fortran, and Python plus an implementation in both CAMB and CLASS.

Finally, for an application, we have used the fitting functions to compute Fisher forecasts for how well a Euclid-like survey will be at constraining the $\mathrm{Hu}-$ Sawicki model. We find that the potential constraints from combining GC and WL from a Euclid-like survey, when including a reasonable amount of nonlinear scales in the forecast, are at the $\Delta f_{R 0} \sim 2 \times 10^{-6}$ level. This is without taking into account baryonic effects in the power spectrum and including massive neutrinos but nevertheless shows the potential constraining power in future surveys when including nonlinear scales.

The same approach as used in this paper can likely be applied to cheaply create emulators for other nonstandard gravity models, possibly in conjunction with the semianalytical method of Ref. [19].

\section{ACKNOWLEDGMENTS}

K. K. and H. A.W. are supported by the European Research Council through Grant No. 646702 (CosTesGrav). K. K. is also supported by the UK Science and Technologies Facilities Council Grant No. ST/N000668/1. G. B.Z. is supported by NSFC Grant No. 11673025 and by a Royal Society-Newton Advanced Fellowship. L. L. is supported by a Swiss National Science Foundation Professorship grant (Grant No. 170547). S. C. acknowledges support from CNRS and CNES grants. B. L. acknowledges the support of the UK STFC Consolidated Grants (Grants No. ST/P000541/1 and No. ST/L00075X/1) and Durham University. B. L. is also supported in part by the European Unions Horizon 2020 research and innovation program. M. B. acknowledges support from the Italian Ministry for Education, University and Research (MIUR) through the SIR individual grant SIMCODE (Project No. RBSI14P4IH), from the grant MIUR PRIN 2015 "Cosmology and Fundamental Physics: Illuminating the Dark Universe with Euclid", and from the Grant agreement No. ASI n.I/023/12/0 Attivit relativealla fase B2/C per la missione Euclid.

\footnotetext{
${ }^{3}$ This can be found at https://github.com/HAWinther/Fofr FittingFunction.
} 
[1] R. Scaramella et al., Euclid space mission: A cosmological challenge for the next 15 years, in Statistical Challenges in 21st Century Cosmology (IAU S306), Part of Proceedings of the International Astronomical Union Symposia and Colloquia Vol. 306, edited by A. Heavens, J.-L. Starck, and A. Krone-Martins (Cambridge University Press, 2015), pp. 375-378, ISBN 9781107078567.

[2] Ž. Ivezić, S. M. Kahn, J. A. Tyson, B. Abel, E. Acosta, R. Allsman, D. Alonso, Y. AlSayyad, S. F. Anderson, J. Andrew and et al., LSST: From science drivers to reference design and anticipated data products, Astrophys. J. 873, 111 (2019).

[3] K. Koyama, Cosmological tests of modified gravity, Rep. Prog. Phys. 79, 046902 (2016).

[4] T. Clifton, P. G. Ferreira, A. Padilla, and C. Skordis, Modified gravity and cosmology, Phys. Rep. 513, 1 (2012).

[5] R. E. Smith, J. A. Peacock, A. Jenkins, S. D. M. White, C. S. Frenk, F. R. Pearce, P. A. Thomas, G. Efstathiou, and H. M. P. Couchman, Stable clustering, the halo model and non-linear cosmological power spectra, Mon. Not. R. Astron. Soc. 341, 1311 (2003).

[6] R. Takahashi, M. Sato, T. Nishimichi, A. Taruya, and M. Oguri, Revising the Halofit model for the nonlinear matter power spectrum, Astrophys. J. 761, 152 (2012).

[7] A. J. Mead, J. A. Peacock, C. Heymans, S. Joudaki, and A. F. Heavens, An accurate halo model for fitting non-linear cosmological power spectra and baryonic feedback models, Mon. Not. R. Astron. Soc. 454, 1958 (2015).

[8] A. J. Mead, C. Heymans, L. Lombriser, J. A. Peacock, O. I. Steele, and H. A. Winther, Accurate halo-model matter power spectra with dark energy, massive neutrinos and modified gravitational forces, Mon. Not. R. Astron. Soc. 459, 1468 (2016).

[9] A. Lewis and S. Bridle, Cosmological parameters from CMB and other data: A Monte Carlo approach, Phys. Rev. D 66, 103511 (2002).

[10] J. Lesgourgues, The cosmic linear anisotropy solving system (CLASS) I: Overview, arXiv:1104.2932.

[11] E. Lawrence, K. Heitmann, M. White, D. Higdon, C. Wagner, S. Habib, and B. Williams, The Coyote Universe. III. Simulation suite and precision emulator for the nonlinear matter power spectrum, Astrophys. J. 713, 1322 (2010).

[12] K. Heitmann, E. Lawrence, J. Kwan, S. Habib, and D. Higdon, The Coyote Universe extended: Precision emulation of the matter power spectrum, Astrophys. J. 780, 111 (2014).

[13] L. Casarini, S. A. Bonometto, E. Tessarotto, and P.-S. Corasaniti, Extending the Coyote emulator to dark energy models with standard $w_{0}-w_{a}$ parametrization of the equation of state, J. Cosmol. Astropart. Phys. 08 (2016) 008.

[14] M. Knabenhans, J. Stadel, S. Marelli, D. Potter, R. Teyssier, L. Legrand, A. Schneider, B. Sudret, L. Blot, S. Awan, C. Burigana, C.S. Carvalho, H. Kurki-Suonio, and G. Sirri (Euclid Collaboration), Euclid preparation: II. The EuclidEmulator-A tool to compute the cosmology dependence of the nonlinear matter power spectrum, Mon. Not. R. Astron. Soc. 484, 5509 (2019).

[15] G.-B. Zhao, Modeling the nonlinear clustering in modified gravity models. I. A fitting formula for the matter power spectrum of $\mathrm{f}(\mathrm{R})$ gravity, Astrophys. J. Suppl. Ser. 211, 23 (2014).

[16] A. Hojjati, L. Pogosian, and G.-B. Zhao, Testing gravity with CAMB and CosmoMC, J. Cosmol. Astropart. Phys. 08 (2011) 005.

[17] W. Hu and I. Sawicki, Models of $f(R)$ cosmic acceleration that evade solar system tests, Phys. Rev. D 76, 064004 (2007).

[18] S. Casas, L. Amendola, M. Baldi, V. Pettorino, and A. Vollmer, Fitting and forecasting coupled dark energy in the non-linear regime, J. Cosmol. Astropart. Phys. 01 (2016) 045.

[19] M. Cataneo, L. Lombriser, C. Heymans, A. Mead, A. Barreira, S. Bose, and B. Li, On the road to per-cent accuracy: Nonlinear reaction of the matter power spectrum to dark energy and modified gravity, Mon. Not. R. Astron. Soc. 488, 2121 (2019).

[20] D. Huterer and M. Takada, Calibrating the nonlinear matter power spectrum: Requirements for future weak lensing surveys, Astropart. Phys. 23, 369 (2005).

[21] A. P. Hearin, A. R. Zentner, and Z. Ma, General requirements on matter power spectrum predictions for cosmology with weak lensing tomography, J. Cosmol. Astropart. Phys. 04 (2012) 034.

[22] A. Schneider, R. Teyssier, D. Potter, J. Stadel, J. Onions, D. S. Reed, R. E. Smith, V. Springel, F. R. Pearce, and R. Scoccimarro, Matter power spectrum and the challenge of percent accuracy, J. Cosmol. Astropart. Phys. 04 (2016) 047.

[23] N. E. Chisari, M. L. A. Richardson, J. Devriendt, Y. Dubois, A. Schneider, A. M. C. Le Brun, R. S. Beckmann, S. Peirani, A. Slyz, and C. Pichon, The impact of baryons on the matter power spectrum from the Horizon-AGN cosmological hydrodynamical simulation, Mon. Not. R. Astron. Soc. 480, 3962 (2018).

[24] A. Schneider and R. Teyssier, A new method to quantify the effects of baryons on the matter power spectrum, J. Cosmol. Astropart. Phys. 12 (2015) 049.

[25] H. A. Winther, F. Schmidt, A. Barreira, C. Arnold, S. Bose, C. Llinares, M. Baldi, B. Falck, W. A. Hellwing, K. Koyama, B. Li, D. F. Mota, E. Puchwein, R. E. Smith, and G.-B. Zhao, Modified gravity N-body code comparison project, Mon. Not. R. Astron. Soc. 454, 4208 (2015).

[26] M. Cautun, E. Paillas, Y.-C. Cai, S. Bose, J. Armijo, B. Li, and N. Padilla, The Santiago-Harvard-Edinburgh-Durham void comparison-I. SHEDding light on chameleon gravity tests, Mon. Not. R. Astron. Soc. 476, 3195 (2018).

[27] B. Li, G.-B. Zhao, R. Teyssier, and K. Koyama, ECOSMOG: An Efficient COde for Simulating MOdified Gravity, J. Cosmol. Astropart. Phys. 01 (2012) 051.

[28] C. Giocoli, M. Baldi, and L. Moscardini, Weak lensing light-cones in modified gravity simulations with and without massive neutrinos, Mon. Not. R. Astron. Soc. 481, 2813 (2018).

[29] E. Puchwein, M. Baldi, and V. Springel, Modified-gravityGADGET: A new code for cosmological hydrodynamical simulations of modified gravity models, Mon. Not. R. Astron. Soc. 436, 348 (2013). 
[30] S. Colombi, A. Jaffe, D. Novikov, and C. Pichon, Accurate estimators of power spectra in N-body simulations, Mon. Not. R. Astron. Soc. 393, 511 (2009).

[31] B. Li, W. A. Hellwing, K. Koyama, G.-B. Zhao, E. Jennings, and C. M. Baugh, The non-linear matter and velocity power spectra in $\mathrm{f}(\mathrm{R})$ gravity, Mon. Not. R. Astron. Soc. 428, 743 (2013).

[32] C. Arnold, P. Fosalba, V. Springel, E. Puchwein, and L. Blot, The modified gravity lightcone simulation project I: Statistics of matter and halo distributions, Mon. Not. R. Astron. Soc. 483, 790 (2019).

[33] H. A. Winther, K. Koyama, M. Manera, B. S. Wright, and G.-B. Zhao, COLA with scale-dependent growth: Applications to screened modified gravity models, J. Cosmol. Astropart. Phys. 08 (2017) 006.

[34] L. Lombriser, K. Koyama, and B. Li, Halo modelling in chameleon theories, J. Cosmol. Astropart. Phys. 03 (2014) 021 .

[35] K. Koyama, A. Taruya, and T. Hiramatsu, Nonlinear evolution of the matter power spectrum in modified theories of gravity, Phys. Rev. D 79, 123512 (2009).

[36] L. Lombriser, B. Li, K. Koyama, and G.-B. Zhao, Modeling halo mass functions in chameleon $\mathrm{f}(\mathrm{R})$ gravity, Phys. Rev. D 87, 123511 (2013).
[37] L. Lombriser, Constraining chameleon models with cosmology, Ann. Phys. (Amsterdam) 526, 259 (2014).

[38] M. Baldi, F. Villaescusa-Navarro, M. Viel, E. Puchwein, V. Springel, and L. Moscardini, Cosmic degeneracies-I. Joint N-body simulations of modified gravity and massive neutrinos, Mon. Not. R. Astron. Soc. 440, 75 (2014).

[39] D. J. Eisenstein and W. Hu, Baryonic Features in the matter transfer function, Astrophys. J. 496, 605 (1998).

[40] D. J. Eisenstein and W. Hu, Power spectra for cold dark matter and its variants, Astrophys. J. 511, 5 (1999).

[41] D. Alonso, E. Bellini, P. G. Ferreira, and M. Zumalacárregui, Observational future of cosmological scalar-tensor theories, Phys. Rev. D 95, 063502 (2017).

[42] R. Laureijs et al., Euclid definition study report, arXiv: 1110.3193.

[43] S. Casas, M. Kunz, M. Martinelli, and V. Pettorino, Linear and non-linear modified gravity forecasts with future surveys, Phys. Dark Universe 18, 73 (2017).

[44] J. Dossett, B. Hu, and D. Parkinson, Constraining models of $\mathrm{f}(\mathrm{R})$ gravity with Planck and WiggleZ power spectrum data, J. Cosmol. Astropart. Phys. 03 (2014) 046.

[45] C. Arnold, P. Fosalba, V. Springel, E. Puchwein, and L. Blot, The modified gravity light-cone simulation project-I. Statistics of matter and halo distributions, Mon. Not. R. Astron. Soc. 483, 790 (2019). 